\title{
Emotional Well-Being Is Impaired in Hidradenitis Suppurativa Patients
}

\author{
Aditi Senthilnathan $^{\mathrm{a}}$ Sree S. Kolli ${ }^{\mathrm{a}}$ Leah A. Cardwell ${ }^{\mathrm{a}}$ Irma M. Richardson ${ }^{\mathrm{a}}$ \\ Steven R. Feldman ${ }^{\mathrm{a}-\mathrm{c}}$ Rita O. Pichardo ${ }^{\mathrm{a}}$ \\ ${ }^{a}$ Center for Dermatology Research, Department of Dermatology, Wake Forest School of Medicine, Winston-Salem, \\ NC, USA; 'bepartment of Pathology, Wake Forest School of Medicine, Winston-Salem, NC, USA; 'Department of \\ Public Health Sciences, Wake Forest School of Medicine, Winston-Salem, NC, USA
}

\author{
Keywords \\ Hidradenitis suppurativa - Positive and Negative Affect \\ Schedule · Depression - Quality of life · \\ Emotional well-being
}

\begin{abstract}
Background: Hidradenitis suppurativa (HS) is a chronic inflammatory condition. Objective: To measure emotional well-being in $\mathrm{HS}$ patients and compare to other populations, assess if there is an effect of disease severity on emotional well-being, and determine if emotional well-being is correlated with quality of life and depression. Methods: A total of $153 \mathrm{HS}$ subjects were recruited, and 66 Positive and Negative Affect Schedule (PANAS) surveys assessing emotional wellbeing were completed. Norms for comparison populations (disease-free undergraduates and adults) were used. A validated self-assessment tool was used to determine disease severity. Subjects completed the Patient Health Questionnaire-9 (PHQ-9) and Dermatology Life Quality Index (DLQI) to examine depression and quality of life, respectively. Results: HS subjects had lower positive affect than undergraduates and higher negative affect than adults. There were no differences in PANAS scores by Hurley stage $(p>0.05)$. PANAS
\end{abstract}

scores for $\mathrm{HS}$ subjects correlated with worse quality of life $(r=$ $0.66, p<0.00001)$ and depression $(r=0.74, p<0.00001)$. Conclusion: $\mathrm{HS}$ patients have poor emotional well-being. PANAS scores correlated with worse quality of life and depression. Providing appropriate resources and treatments may be beneficial for HS patients.

(c) 2019 S. Karger AG, Basel

\section{Introduction}

Hidradenitis suppurativa (HS) is a chronic inflammatory condition characterized by recurrent abscesses and scarring. The disfiguring lesions can lead to stigma and embarrassment, lowering self-esteem and creating a negative outlook in patients. The Positive and Negative Affect Schedule (PANAS) scale is a validated and reliable measure that assesses emotional well-being and has the potential to detect mental illnesses [1]. We assessed emotional wellbeing in HS patients using this tool. We also compared HS PANAS scores to those in other populations, examined the effect of disease severity as measured by Hurley stage on emotional well-being, and assessed correlation of PANAS scores with depression and quality of life.

\section{KARGER}

(c) 2019 S. Karger AG, Basel

E-Mail karger@karger.com

www.karger.com/sad
Sree S. Kolli

Department of Dermatology, Wake Forest School of Medicine

1 Medical Center Boulevard

Winston-Salem, NC 27157-1071 (USA)

E-Mail skolli@wakehealth.edu 
Table 1. PANAS scores for HS compared to undergraduates and adults

\begin{tabular}{lll}
\hline & Positive affect & Negative affect \\
\hline PANAS questionnaire scores in HS subjects & Interested (3) & Distressed (2.6) \\
& Excited (2.7) & Guset $(2.5)$ \\
& Strong (2.8) & Scared (1.5) \\
& Enthusiastic (2.7) & Hostile $(1.9)$ \\
& Proud (2.9) & Irritable (2.8) \\
& Alert (3) & Ashamed $(2.5)$ \\
\hline HS $(n=66)($ mean \pm SD) & Inspired (2.6) & Nervous $(2.4)$ \\
\hline Undergraduates $(n=1,521)($ mean \pm SD) & Determined (3.4) & Afraid $(1.9)$ \\
\hline Adults $(n=328)($ mean \pm SD) & Attentive (3.1) & $22.0 \pm 10.1^{\mathrm{b}}$ \\
\hline
\end{tabular}

Values in parentheses are means. ${ }^{a}$ HS subjects had lower positive feelings than undergraduates $(p<0.01)$. ${ }^{\mathrm{b}}$ HS subjects had higher negative feelings than adults $(p<0.01)$.

\section{Methods}

A total of 153 subjects ( 30 in clinic and 123 through mail) clinically diagnosed with HS (ICD-10 code: L73.2) were recruited either in person at the Wake Forest Baptist Health (WFBH) dermatology clinic or via mail surveys between June to September 2018. HS patients identified by the electronic medical record were surveyed. Subjects over 18 years of age diagnosed with HS by a dermatologist at the WFBH dermatology clinic were eligible to participate. Subjects younger than 18 years of age or those who did not have a mailing address on file were excluded.

Emotional well-being over the past week was assessed in 66 subjects (27 in clinic and 39 through mail) using the PANAS. The PANAS is a self-reported questionnaire comprised of two 10-item scales that measure positive and negative affect. The positive items are words such as "attentive," "alert," and "inspired." The negative items are words such as "hostile," "irritable," and "upset." Each item is rated on a 5-point scale from 1 (not at all) to 5 (very much) [2]. Norms of positive and negative affect have been established from a nonclinical undergraduate sample; norms from a large-scale clinical sample have not been reported for this tool. Adequate internal consistency has been established; the original study found an average Cronbach's alpha of 0.88 for the positive affect items and 0.87 for the negative affect items. Adequate test-retest reliability for the PANAS has been established [3]. In our analysis, PANAS scores of HS patients were compared to the undergraduate sample used to establish PANAS norms and to an adult sample [2] using two-sample $t$ tests.

The overall response rate was $90 \%$ for the clinic population and $32 \%$ for the mail survey. Subjects reported their disease severity using a validated HS Self-Assessment (HSSA) tool that assigned a Hurley stage [4]. Subjects also completed the Patient Health Questionnaire-9 (PHQ-9) and Dermatology Life Quality Index (DLQI) to examine depression and quality of life, respectively, and their correlation with PANAS scores (using Pearson correlation coefficient) [5, 6]. Student $t$ tests and ANOVA were used for between group comparisons.

\section{Results}

The average age of respondents was 39 years, $89 \%$ were female, and $58 \%$ identified as African American. Nonresponders consisted of 87 patients (57\%) with comparable demographics (mean age 36 years, $80 \%$ female, and $38 \%$ African American).

HS subjects had lower positive affect than a sample of undergraduates and higher negative affect than a sample of adults (Table 1). The mean difference between PANAS positive affect scores in HS subjects and a sample of undergraduates was $-3.7(1.2)$ with a $95 \%$ confidence interval (CI) of $(-6.1$ to -1.4$)$ and a $p$ values of 0.0026 . The mean difference between these samples for negative affect scores was $1.6(1.3)$ with a $95 \%$ CI of $(-0.9$ to 4.1$)$ and a $p$ value of 0.21 . The mean difference between PANAS positive affect scores in HS subjects and a sample of adults was -2.4 (1.2) with $95 \%$ CI ( -4.9 to 0.1$)$ and a $p$ value of 0.06 . The mean difference between PANAS negative affect scores in HS subjects and a sample of adults was 4.0 (1.3) with $95 \%$ CI (1.4 to 6.6) and a $p$ value of 0.003 .

There were no differences in PANAS scores by Hurley stage $(p>0.05)$. PANAS scores for HS subjects correlated with worse quality of life $(r=0.66, p<0.00001)$ and depression ( $r=0.74, p<0.00001$; Fig. 1$)$. 


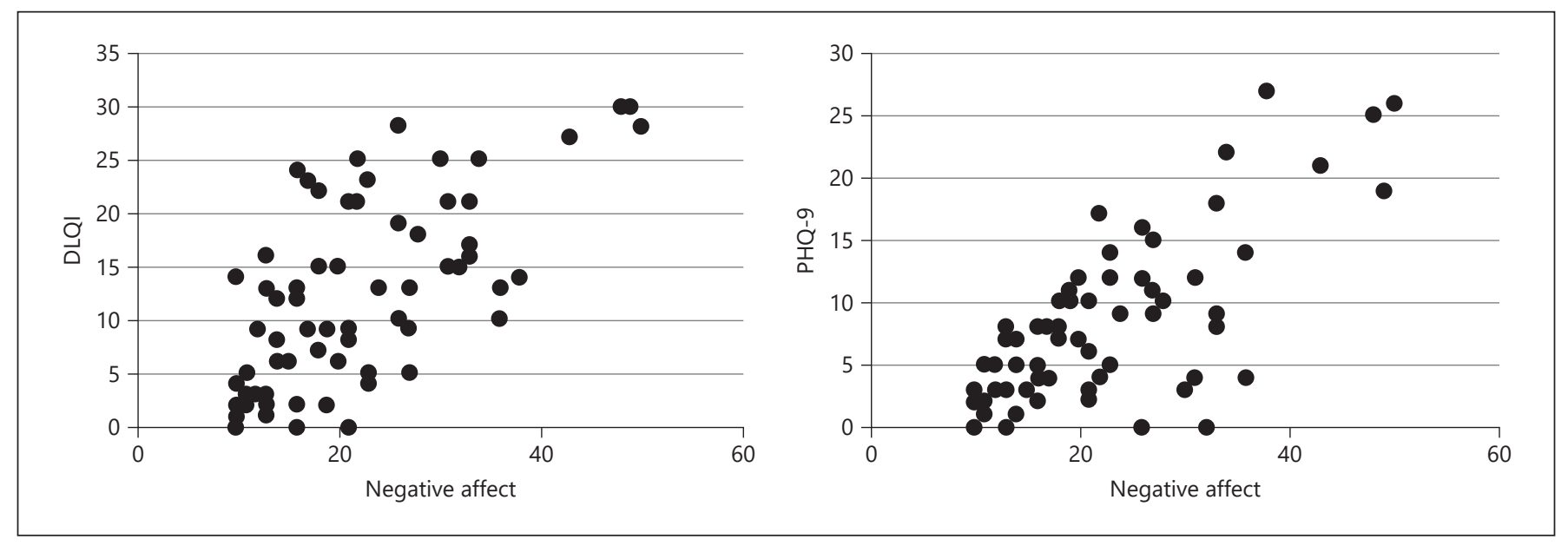

Fig. 1. PANAS scores for HS correlate with quality of life and depression. Higher negative affect scores indicate worse emotional well-being. Higher DLQI and PHQ-9 scores indicate worse quality of life and depressive symptoms, respectively. Negative affect moderately correlates with worse quality of life $(r=0.66, p<0.00001)$ and depression $(r=0.74, p<0.00001)$. DLQI, Dermatology Life Quality Index; PHQ-9, Patient Health Questionnaire-9.

\section{Discussion}

The level of negative feelings is high in HS patients compared to healthy adults. The lack of variation in PANAS scores by Hurley stage suggests that even mild HS contributes to poor emotional well-being. Although disease severity was self-reported, the measure was validated with physician ratings of severity. Greater negative affect correlated with worse quality of life and more depressive symptoms. Our sample size was small, but the effect sizes were large enough to detect significant associations. Another limitation is the potential for differences other than HS between our population and the comparator populations. Our findings appear generalizable given the comparable demographics between survey responder and nonresponder populations.

Providing greater emotional support and appropriate resources for HS patients could be beneficial in addressing a diminished quality of life as these patients are at an increased risk of suicide $[7,8]$. Treatments that give a high degree of clearing are needed given that even mild HS has a large impact on patients.

\section{Statement of Ethics}

Approval from the Institutional Review Board (IRB; approval $\# 00049730$ ) was obtained prior to initiation of the study. The study is registered at ClinicalTrials.gov, NCT03572738. All procedures performed in studies involving human participants were in accordance with the ethical standards of the institutional and/or na- tional research committee and with the 1964 Helsinki declaration and its later amendments or comparable ethical standards. Informed consent was obtained from all individual participants included in the study.

\section{Disclosure Statement}

Dr. Steven R. Feldman is a speaker for Janssen and Taro. He is a consultant and speaker for Galderma, Stiefel/GlaxoSmithKline, Abbott Labs, and Leo Pharma Inc. Dr. Feldman has received grants from Galderma, Janssen, Abbott Labs, Amgen, Stiefel/GlaxoSmithKline, Celgene, and Anacor. He is a consultant for Amgen, Baxter, Caremark, Gerson Lehrman Group, Guidepoint Global, Hanall Pharmaceutical Co. Ltd., Kikaku, Lilly, Merck \& Co. Inc., Merz Pharmaceuticals, Mylan, Novartis Pharmaceuticals, Pfizer Inc., Qurient, Suncare Research, and Xenoport. He is on an advisory board for Pfizer Inc. Dr. Feldman is the founder and holds stock in Causa Research and holds stock and is majority owner of Medical Quality Enhancement Corporation. He receives Royalties from UpToDate and Xlibris. Dr. Rita O. Pichardo has received consulting support from Abbvie. Sree S. Kolli, Aditi Senthilnathan, Leah A. Cardwell, and Irma M. Richardson have no conflicts to disclose.

\section{Author Contributions}

All named authors meet the International Committee of Medical Journal Editors (ICMJE) criteria for authorship for this article, take responsibility for the integrity of the work as a whole, and have given their approval for this version to be published. 


\section{References}

1 Watson D, Clark LA, Tellegen A. Development and validation of brief measures of positive and negative affect: the PANAS scales. J Pers Soc Psychol. 1988 Jun;54(6):1063-70.

2 Watson D, Clark LA. The PANAS-X: Manual for the Positive and Negative Affect ScheduleExpanded Form. Ames: The University of Iowa; 1994

3 Crawford JR, Henry JD. The positive and negative affect schedule (PANAS): construct validity, measurement properties and norma- tive data in a large non-clinical sample. Br J Clin Psychol. 2004 Sep;43(Pt 3):245-65.

4 Senthilnathan A, Kolli SS, Cardwell LA, Richardson I, Feldman SR, Pichardo RO. Validation of hidradenitis suppurativa self-assessment tool. J Cutan Med Surg. 2019 Jul/Aug; 23(4):388-90.

5 Kroenke K, Spitzer RL, Williams JB. The PHQ-9: validity of a brief depression severity measure. J Gen Intern Med. 2001 Sep;16(9): 606-13.
6 Finlay AY, Khan GK. Dermatology Life Quality Index (DLQI) - a simple practical measure for routine clinical use. Clin Exp Dermatol. 1994 May;19(3):210-6.

7 Tiri H, Huilaja L, Jokelainen J, Timonen M, Tasanen K. Women with Hidradenitis Suppurativa Have an Elevated Risk of Suicide. J Invest Dermatol. 2018 Dec;138(12):2672-4.

8 Thorlacius L, Cohen AD, Gislason GH, Jemec GB, Egeberg A. Increased Suicide Risk in $\mathrm{Pa}-$ tients with Hidradenitis Suppurativa. J Invest Dermatol. 2018 Jan;138(1):52-7. 